\title{
OPEN Associations between smoking and blood-group, and the risk of dyslipidaemia amongst French women
}

\author{
C. J. MacDonald ${ }^{1,2}$, A. L. Madika ${ }^{1,2,3}$, G. Severi ${ }^{1,2,4}$, A. Fournier ${ }^{1,2}$ \& M. C. Boutron-Ruault ${ }^{1,2}$
}

Dyslipidaemia is a major risk factor for cardio-vascular disease, as it promotes atherosclerosis. While cross-sectional studies have identified higher serum cholesterol amongst individuals with the A blood group, there is less evidence from prospective studies whether this translates into a higher risk of dyslipidaemia that requires treatment, nor if this genetic factor interacts with smoking status. This study aimed to prospectively determine potential associations between smoking, ABO blood groups, and risk of incident dyslipidaemia requiring treatment, and to assess associations over strata of blood $A B O$ group. We assessed associations between blood $A B O$ group, smoking and dyslipidaemia in 74,206 women participating in the E3N cohort. We included women who did not have cardiovascular disease at baseline. Logistic regression was used to determine associations between ABO group, smoking and prevalent dyslipidaemia at baseline. Cox proportional hazard models were then used to determine if blood $A B O$ group and smoking were associated with the risk of incident dyslipidaemia, amongst women free of dyslipidaemia at baseline. At baseline 28,281 women with prevalent dyslipidaemia were identified. Compared to the O-blood group, the non-O blood group was associated higher odds of with prevalent dyslipidaemia $\left(O R_{n o n-0}=1.09\right.$ [1.06: 1.13]). Amongst the women free of dyslipidaemia at baseline, 6041 incident cases of treated dyslipidaemia were identified during 454,951 person-years of follow-up. The non-O blood groups were associated with an increased risk of dyslipidaemia when compared to the O-group $\left(\mathrm{HR}_{\text {non-o }}=1.16\right.$ [1.11: 1.22]), specifically the $A$ bloodgroup $\left(\mathrm{HR}_{\mathrm{A}}=1.18\right.$ [1.12: 1.25]). Current smokers were associated with an increased risk of incident dyslipidaemia (HR ${ }_{\text {smokers }}=1.27$ [1.16: 1.37]), compared to never-smokers. No evidence for effect modification between smoking and $A B O$ blood group was observed ( $p$-effect modification $=0.45$ ), although the highest risk was observed among $A B$ blood group women who smoked (HR =1.76 [1.22: 2.55]). In conclusion, the non-O blood groups, specifically the A group were associated with an increased risk of dyslipidaemia. Current smokers were associated with a $30 \%$ increased risk of dyslipidaemia. These results could aid in personalised approaches to the prevention of cardiovascular risk-factors.

\author{
Abbreviations \\ BMI Body mass index \\ MHT Menopausal hormone therapy
}

Dyslipidaemia is the main risk-factor for heart disease ${ }^{1}$, and can lead to the formation of atherosclerotic plaques $^{2}$ which can narrow the coronary arteries, causing life-threatening blockages. High low-density lipoprotein (LDL) cholesterol levels can also lead to endothelial dysfunction and oxidative stress ${ }^{3}$, both additional risk-factors for heart disease ${ }^{4}$.

\footnotetext{
${ }^{1}$ INSERM (Institut National de La Santé Et de La Recherche Médicale) U1018, Center for Research in Epidemiology and Population Health (CESP), Institut Gustave Roussy, 114, rue Edouard Vaillant, 94805 Villejuif cedex, France. ${ }^{2}$ Université Paris-Saclay, Université Paris-Sud, Villejuif, France. ${ }^{3}$ Université de Lille, CHU Lille, EA 2694 - Santé Publique : Épidémiologie et Qualité des Soins, 59000 Lille, France. ${ }^{4}$ Department of Statistics, Computer Science and Applications "G. Parenti", University of Florence, Florence, Italy. ${ }^{\varpi}$ email: marie-christine.boutron@ gustaveroussy.fr
} 
The A blood group has been found to be associated with higher serum cholesterol levels ${ }^{5}$ since the observation of higher cholesterol among non-O blood group males in the prospective Framingham Heart study of 5209 adults, and amongst 10,000 Israeli government employees in the $1970 \mathrm{~s}^{6,7}$, and it has been suggested that associations between non-O groups and cardiovascular disease are mediated by serum cholesterol levels ${ }^{8}$. The non-O blood groups, most commonly the A-group, have been identified as a genetic risk factor for cardiovascular disease in meta-analyses of prospective and retrospective studies ${ }^{9-11}$ and have long been associated with higher rates of ischemic heart disease. Similarly, smoking is a major risk-factor for dyslipidaemia ${ }^{12,13}$ and further cardiovascular disease ${ }^{14}$, Current smokers have been shown to have higher serum cholesterol levels than non-smokers, including higher low-density lipoprotein (LDL) levels, but also low high-density lipoprotein (HDL) concentrations ${ }^{15}$, 16. Smoking is also associated with increased oxidation of LDL cholesterol, which may explain associations with atherosclerosis ${ }^{7}$, and it has been shown to interact with ABO blood group in other vascular diseases including thromboembolism ${ }^{17}$. Studies have not assessed if interactions with blood group are present when considering dyslipidaemia, despite both factors leading to abnormal lipid profiles. If current smokers with non-O blood group were found to be at an even higher risk of dyslipidaemia, this could aid in the search for personalised preventative approaches for cardiovascular risk factors.

We aimed to investigate if non-O blood group and smoking were associated with an increased risk of dyslipidaemia in a large prospective cohort of French women, and to investigate if there was evidence for effect modification between the two risk-factors, hypothesising that stronger associations may be observed among non-O group women who smoked, due to higher lipid levels.

\section{Methods}

All methods were carried out in accordance with the relevant guidelines and regulations.

The E3N cohort. The Etude Épidémiologiquede femmes de la Mutuelle Générale de l'Education (E3N) is a French prospective cohort started in 1990 comprising 98,995 women aged 40-65 years at baseline and insured by the Mutuelle Générale de l'Education Nationale (MGEN), a health insurance plan for employees of the French education system and their families ${ }^{18}$. The cohort received ethical approval from the French National Commission for Computerized Data and Individual Freedom (Commission Nationale Informatique et Libertés), and all participants in the study signed an informed consent form. Participants returned mailed questionnaires on lifestyle information and disease occurrence every 2 to 3 years (1990, 1992, 1993, 1995, 1997, 2000, 2002, 2005, 2008, 2011, and 2014), resulting in 11 questionnaires (Q1-11). The average response rate at each questionnaire cycle was $83 \%$, and the total loss to follow-up was 3\%. Furthermore, for each participant, the MGEN health insurance plan provided data that included all drug reimbursements since January 1, 2004.

Assessment of dyslipidaemia. In the first two questionnaires sent in 1990 and 1992 (Q1 \& Q2), participants were asked if they were undergoing treatment for raised blood cholesterol, and to provide serum cholesterol values if available in mmol/L. In Q6, Q7 and Q8 participants were asked if their previous cholesterol measurement was abnormal, and to provide serum cholesterol values. In Q9, Q10 and Q11, participants were asked to report if they were undergoing treatment for high cholesterol.

The drug reimbursement database allowed us to identify reimbursement for lipid lowering medications in 2004 (identified using Anatomical Therapeutic Chemical Classification System C10A and C10B). We considered incident cases of dyslipidaemia as those reporting high or treated blood cholesterol, confirmed by a reimbursement for lipid lowering medications at the same time as the questionnaire (+-1 year, except for Q6 which considered reimbursements at 2004).

Cases in this study were defined as either: self-reported 'abnormal or high cholesterol requiring treatment', or self-reported serum cholesterol $>=6.6 \mathrm{mmol} / \mathrm{L}$ ('self-reported dyslipidaemia'). We considered an additional criteria, requiring identification of a lipid-lowering medication during the period corresponding to the questionnaire that dyslipidaemia was reported ('treated dyslipidaemia'). Due to the significant number of women not reporting cholesterol values, we did not consider cholesterol in $\mathrm{mmol} / \mathrm{L}$ as the outcome.

Assessment of blood $\mathrm{ABO}$ group. Participants were asked to report their blood $\mathrm{ABO}$ group in the first questionnaire as $O, A, B$, or $A B$, as well as their Rhesus group as positive or negative.

Assessment of smoking status. Smoking status was considered at every questionnaire, and was based on self-reports. Subjects were classified as never, former or current smokers. Past or current smokers of less than one cigarette per day (occasional smokers) were considered non-smokers.

Assessment of covariates. Height and weight were self-reported at each questionnaire and used to calculate body mass index $\left(\mathrm{BMI}\left(\mathrm{kg} / \mathrm{m}^{2}\right)\right.$ ). Family history of cardiovascular disease (stroke or myocardial infarction) was based on self-reports. Education level was self-reported and used as a proxy for social class. Total physical activity was self-reported in Q5, and detailed time spent undergoing various activities (such as walking, housework, sports). Total Metabolic equivalents (MET-hours) were estimated for each individual using the compendium of physical activity. Hypertension was self-reported and verified by cross-correlation with the drug reimbursement database, to confirm that the participants were undergoing treatment. Use of menopausal hormone therapy (MHT) was self-reported during follow-up. The complete history of HT use was established using data from all the questionnaires ${ }^{19}$. Among postmenopausal women, age at menopause was defined as either (in decreasing order of priority) age at last menstrual period, age at bilateral oophorectomy, self-reported age at 
menopause, age at start of MHT, or the age at the start of menopausal symptoms. If unavailable, the median age at menopause for the cohort (51 years for natural menopause, 47 years for artificial menopause) was imputed.

Study population. From the 98,995 women of the E3N study, we excluded women for whom there was no information on potential dyslipidaemia $(n=14,652)$, blood group $(n=7189)$, at or prior to Q6, women who died, or did not provide data on covariates prior to Q7 $(\mathrm{N}=2948)$, resulting in 74,206 women being included.

Statistical analysis. Two main analyses were performed. We first considered associations between prevalent self-reported dyslipidaemia at baseline (Q7) and the characteristics of the cohort, including blood ABO group. Secondly, we considered only incident cases after Q7 in a time-to-event model, and excluded all prevalent cases considered in the first analysis.

Baseline associations. We assessed associations between baseline variables at Q7 and self-reported prevalent dyslipidaemia (yes/no) amongst women alive at Q7 using a logistic regression model, with prevalent dyslipidaemia as a binary variable. Results were interpreted as odd ratio for prevalent dyslipidaemia, with $95 \%$ confidence interval.

Incident dyslipidaemia cases. For the analysis of incident dyslipidaemia cases, we considered a time-to-event model, and excluded all prevalent cases from this analysis, resulting in a sample of 45,925. Cases were initially considered as all incident self-reports of abnormal blood cholesterol, and then only self-report verified reimbursement for lipid-lowering medications. Participants contributed person years to the analysis from inclusion until the participant reported dyslipidaemia, the participant's death, loss to follow up, or the administrative end of the study period (June 2014).

$\mathrm{ABO}$ group was considered an exposure in Cox proportional hazard models, with the $\mathrm{O}$-group as reference. $\mathrm{ABO}$ group was considered as $O /$ non- $O$, then as $O, A, B$, and $A B$, and also Rhesus + or -. Smoking status at baseline was then considered as the exposure, grouped as current smoker, ex-smoker, and never smokers, with never smokers as the reference group.

Models were initially adjusted with age as the timeline, followed by potential confounders BMI (continuous), physical activity (continuous), education (university degree or not), treated hypertension (yes/no), treated diabetes (yes/no), family history of CVD (yes/no), ever use of menopausal hormone therapy (yes/no), age at menopause. We assessed a-priori effect-modification between $\mathrm{ABO}$ group and smoking status by including the joint term in the main model. The $\mathrm{p}$-value for this term was used to determine if effect-modification was likely.

Data is presented as mean (standard deviation) for continuous variables, median (inter-quartile range) for non-normal data and \% for categorical variables. All analysis was conducted using the statistical software R, version 3.5 , with the survival package.

Ethical approval and consent to participate. The cohort received ethical approval from the French National Commission for Computerized Data and Individual Freedom (Commission Nationale Informatique et Libertés), and all participants in the study signed an informed consent.

\section{Results}

At inclusion, there were 70,177 women included in the study. Non-O blood group was reported in $56.7 \%$ of women. The mean age was 59.2 years old, BMI was $23.8 \mathrm{~kg} / \mathrm{m}^{2}$, and $90.5 \%$ of women were menopausal. Full cohort characteristics are presented in Table 1.

Associations between prevalent dyslipidaemia and baseline characteristics. At baseline 17,223 women reported prevalent dyslipidaemia. Cholesterol values were provided by $42,358(57.1 \%)$ participants, and values were higher among women reporting dyslipidaemia, compared to those who did not (6.2 vs $5.4 \mathrm{mmol} / \mathrm{L})$. Among women reporting dyslipidaemia, cholesterol values were on average lower if they were reimbursed for lipid-lowering drugs (6.4 vs $5.9 \mathrm{mmol} / \mathrm{L}$ ). Cholesterol values were similar among women not reporting dyslipidaemia, regardless of statin reimbursement (data not shown).

Using a multivariate logistic regression model, it was observed that those with prevalent dyslipidaemia were more likely to be of non-O blood group ( $\mathrm{OR}=1.17$ [1.13: 1.21], Table 1) compared to those without dyslipidaemia. Women with dyslipidaemia at baseline were also more likely to be older, have a higher BMI, more likely to have familial history of cardiovascular disease, have diabetes or hypertension, and were less likely to be current smokers (Table 1).

Associations between blood ABO group, and incident dyslipidaemia during follow-up. Amongst the 45,925 women free of dyslipidaemia at baseline, 8370 reported incident high blood cholesterol during 454,951 person years, 6041 (72.2\%) of whom were verified by reimbursement for lipid-lowering medications. This corresponded to a rate of 18.4/1,000 PY. No major differences in these women's characteristics were observed according to blood-group (supplementary Table 1).

Compared to the $\mathrm{O}$ group, the non-O groups were associated with an increased risk of incident dyslipidaemia $\left(\mathrm{HR}_{\text {non-O }}=1.14\right.$ [1.10: 1.19], Table 2). Specifically, the $A$ blood group was associated with an increased risk of dyslipidaemia when compared to the O-group $\left(\mathrm{HR}_{\mathrm{A}}=1.18\right.$ [1.13: 1.23], Table 2), independent of other risk factors. Other blood groups were not associated with the risk of dyslipidaemia $\left(\mathrm{HR}_{\mathrm{B}}=1.03\right.$ [0.98: 1.09], $\mathrm{HR}_{\mathrm{AB}}=1.04$ [0.97: 1.12], Table 2). Rhesus group was not associated with the risk of dyslipidaemia. 


\begin{tabular}{|c|c|c|c|c|}
\hline & Whole population $N=74,206$ & No dyslipidaemia $N=45,925$ & Prevalent dyslipidaemia $\mathrm{N}=\mathbf{2 8 , 2 8 1}$ & $\begin{array}{l}\text { Odds ratio between no } \\
\text { dyslipidaemia and prevalent } \\
\text { dyslipidaemia }^{\star}\end{array}$ \\
\hline Cholesterol (mmol/L) & $5.8(1.1)$ & $5.4(0.8)$ & $6.2(1.3)$ & - \\
\hline $\begin{array}{l}\text { Missing data on serum-cholesterol } \\
(\%)\end{array}$ & 42.9 & 48.4 & 34.1 & - \\
\hline $\begin{array}{l}\text { Reimbursed for lipid-lowering } \\
\text { medication (\%) }\end{array}$ & 26.0 & 14.8 & 44.1 & - \\
\hline \multicolumn{5}{|l|}{ Cohort characteristics } \\
\hline Never smoker (\%) & 53.6 & 52.6 & 55.4 & Ref \\
\hline Ex-smoker (\%) & 36.9 & 37.1 & 36.5 & 1.00 [0.97: 1.03] \\
\hline Current smoker (\%) & 9.5 & 10.3 & 8.1 & $0.87[0.82: 0.92]$ \\
\hline Non-O blood group (\%) & 56.8 & 55.9 & 58.3 & 1.09 [1.06: 1.13] \\
\hline Rhesus group + (\%) & 82.9 & 83.0 & 82.6 & 0.98 [0.94: 1.03] \\
\hline Age at baseline (years) & $61.5(6.5)$ & $60.7(6.3)$ & $62.7(6.6)$ & $1.05[1.04: 1.05]$ \\
\hline BMI $\left(\mathrm{kg} / \mathrm{m}^{2}\right)$ & $23.8(3.7)$ & $23.7(3.7)$ & $24.0(3.7)$ & 1.01 [1.00: 1.02] \\
\hline Total physical activity (Mets-h/week) & $65.6(37.8)$ & $61.7(37.6)$ & $63.1(38.0)$ & $1.00[1.00: 1.00]$ \\
\hline $\begin{array}{l}\text { Family history of cardiovascular } \\
\text { disease (\%) }\end{array}$ & 34.7 & 32.6 & 38.2 & $1.30[1.25: 1.34]$ \\
\hline Prevalent hypertension (\%) & 43.4 & 41.1 & 47.1 & 1.11 [1.07: 1.14] \\
\hline Prevalent diabetes (\%) & 3.4 & 2.9 & 4.1 & 1.27 [1.16: 1.38$]$ \\
\hline Education (high-school or higher) & 87.6 & 88.1 & 86.7 & 0.98 [0.93: 1.02$]$ \\
\hline Menopausal at baseline & 89.6 & 88.0 & 92.2 & 1.07 [1.00: 1.15$]$ \\
\hline Ever use of MHT ${ }^{* *}$ (yes or no) & 60.0 & 60.0 & 60.1 & $0.99[0.96: 1.03]$ \\
\hline Age at menopause ${ }^{* *}$ (years) & 50.3 & 50.6 & 50.4 & 0.99 [0.98: 0.99] \\
\hline
\end{tabular}

Table 1. Associations between baseline characteristics and prevalent dyslipidaemia, odds ratio calculated using a mutually adjusted logistic regression model. ${ }^{\star}$ Adjusted simultaneously for all variables. ${ }^{*}$ Amongst menopausal women. BMI body mass index, $M H T$ menopausal hormone therapy.

\begin{tabular}{|l|l|l|}
\hline Blood group & Self-reported dyslipidaemia (cases=8370 $)$ & $\begin{array}{l}\text { Self-reported dyslipidaemia + medication } \\
\text { reimbursement (cases=6041) }\end{array}$ \\
\hline$O(n=20,260)$ & Ref & Ref \\
\hline Non-O $(n=25,665)$ & $1.14[1.09: 1.19]$ & $1.16[1.11: 1.22]$ \\
\hline$A(n=19,899)$ & $1.15[0.10: 1.20]$ & $1.18[1.12: 1.25]$ \\
\hline$B(n=4020)$ & $1.06[0.98: 1.15]$ & $1.08[0.98: 1.19]$ \\
\hline$A B(n=1746)$ & $1.14[1.02: 1.27]$ & $1.13[0.98: 1.29]$ \\
\hline Rhesus $+(n=38,118)$ & Ref & Ref \\
\hline Rhesus $-(n=7807)$ & $0.97[0.92: 1.03]$ & $0.97[0.91: 1.03]$ \\
\hline Smoking status & \multicolumn{2}{|l|}{} \\
\hline Never smoker $(n=24,144)$ & Ref & Ref \\
\hline Ex-smoker $(n=17,043)$ & $1.07[1.02: 1.12]$ & $1.04[0.99: 1.10]$ \\
\hline Current smoker $(n=4738)$ & $1.23[1.14: 1.33]$ & $1.27[1.16: 1.37]$ \\
\hline
\end{tabular}

Table 2. Risk of incident dyslipidaemia depending on blood ABO group, hazard ratios (HR) estimated from Cox proportional hazards models. Adjusted for body mass index, physical activity, education level, diabetes, hypertension, family history of cardiovascular disease, use of menopausal hormone therapy, and age at menopause. Age as the timescale.

Compared to never smokers, women who were current smokers were of an increased risk of incident dyslipidaemia, in both age adjusted, and adjusted models (adjusted $\mathrm{HR}_{\text {smokers }}=1.33$ [1.24: 1.43]; $\mathrm{HR}_{\text {ex-smokers }}=1.06$ [1.01: 1.10], Table 2).

Effect modification was not likely between blood group ( $\mathrm{O}$ vs non-O) and smoking ( $\mathrm{p}$ - effect modification $=0.45$ ). Participants with an O blood group who were not smokers reported baseline serum cholesterol values of $5.4 \mathrm{mmol} / \mathrm{L}$ at baseline, compared to $5.5 \mathrm{mmol} / \mathrm{L}$ among A-group participants who smoked. Associations between current smokers and dyslipidaemia were slightly higher amongst participants with a non-O blood group $($ HR smokers = 1.31 [1.16: 1.47], Table 3), compared to those with an O blood group (HR smokers $=1.22$ [1.06: 1.40], Table 3), although the confidence intervals overlapped. Smokers of the AB-group were at the highest risk of dyslipidaemia $(\mathrm{HR}$ smokers $=1.76[1.22: 2.55])$. The adjusted case rate for treated dyslipidaemia amongst 


\begin{tabular}{|c|c|c|c|c|c|}
\hline \multirow[b]{2}{*}{ Blood group } & \multicolumn{5}{|l|}{ Adjusted $^{*} \mathrm{HR}$} \\
\hline & O-Group & Non O-Group $(n=20,260)$ & A $(n=19,899)$ & $B(n=4020)$ & $\mathrm{AB}(n=1746)$ \\
\hline \multicolumn{6}{|l|}{ Smoking status } \\
\hline Never smoker & Ref & Ref & Ref & Ref & Ref \\
\hline Ex-smoker & $0.99[0.91: 1.08]$ & 1.09 [1.01: 1.17] & 1.10 [1.02: 1.17] & 1.10 [0.94: 1.28$]$ & $1.22[0.97: 1.54]$ \\
\hline Current smoker & 1.22 [1.06: 1.40$]$ & 1.31 [1.16: 1.47] & $1.22[1.09: 1.36]$ & $1.21[0.92: 1.58]$ & $1.76[1.22: 2.55]$ \\
\hline
\end{tabular}

Table 3. Risk of incident dyslipidaemia depending on smoking status, stratified by blood ABO group, hazard ratios (HR) estimated from Cox proportional hazards models. ${ }^{\star}$ Adjusted for body mass index, physical activity, education level, diabetes, hypertension, family history of cardiovascular disease, use of menopausal hormone therapy, and age at menopause. Age as the timescale. Considering dyslipidaemia validated by medication reimbursements.

$\mathrm{AB}$ women who smoked was 23.4 cases/1000 PY, compared to 12.8 cases/1000 PY among O-group women who did not smoke.

\section{Discussion}

In this large prospective study, women of non-O blood group, specifically $A$, were at an $18 \%$ higher risk of developing dyslipidaemia, regardless of other risk factors including diet, smoking, physical activity, and BMI. At baseline, prevalent dyslipidaemia was also associated with other metabolic conditions, such as hypertension, diabetes, family history of cardiovascular disease, and a higher BMI. Smoking prevalence was lower amongst participants with prevalent dyslipidaemia, suggesting possible attempts to improve lifestyle factors following a diagnosis of high-cholesterol. During follow-up, current smokers were associated with a near $30 \%$ increased risk of dyslipidaemia compared to non-smokers. No interaction between blood-group and smoking was observed, although risk was slightly higher amongst smokers with an A blood-group.

Previous large prospective studies conducted since the $1970 \mathrm{~s}^{6,7}$, have shown that high concentrations of cholesterol are more common in non-O blood group persons and particularly in A-group people. Interestingly, we observed that only the $A$-group was associated with increased rates of dyslipidaemia, and not the $B$ or $A B$, although this could be explained by lower sample sizes for these women. Similar results have been observed recently in the large prospective UK biobank study $(n=406755)^{20}$, with observations that the $A$-blood group was associated with a $9 \%$ higher risk of hyperlipidaemia when compared to the $O$-group, and $8 \%$ when compared to the $B$-group. Non- $O$ was also a predictor of other diseases such as thrombosis in the UK biobank study ${ }^{20}$, and it was also observed that non-O groups were associated reduced longevity, and with lower rates of hypertension. Another recent Italian cross-sectional study of 5063 blood donors found that the $O$-group was more prevalent amongst centenarians, and the authors suggest that this is due to associations with longevity ${ }^{21}$. Another small prospective study identified that non-O blood group is associated with clinical and subclinical cardiovascular events $^{22}$. Mechanisms between blood-group and cardiovascular diseases are commonly explained via increases in inflammatory markers ${ }^{23-25}$, von Willebrand factor ${ }^{26}$, and a higher cholesterol burden leading to atherosclerosis, and finally to acute cardiovascular events.

Although the exact mechanism in which the blood group allele causes higher serum cholesterol is unclear, studies have shown that risk alleles in ABO group are associated with higher absorption of cholesterol in the intestines ${ }^{27}$, which may be one mechanism in which serum cholesterol is chronically higher, resulting in a higher lifetime cholesterol burden in non-O group people. The non-O variant is considered independent of other genetic factors which could cause familial hypercholesterolemia ${ }^{28}$, such as mutations in the LDL-receptor gene. The A-blood group has previously been associated with a higher risk of coronary artery disease, likely due to associations with serum cholesterol, and previous work has identified that serum cholesterol is a mediator of this relationship ${ }^{8}$.

We did not observe strong effect modification between smoking and blood-group. Smoking has been identified as a factor which alters the blood lipid profile ${ }^{7,15,16}$, and results in increases in LDL-C and decreases in HDL-C. Studies have not assessed differences in cholesterol levels across joint smoking and blood groups, a measurement that could be taken in future studies. We observed no evidence for any statistical effect-modification or interaction, although rates of dyslipidaemia were highest amongst women with an AB blood-group who smoked.

The strengths of this study include its prospective design, large number of participants and cases, control for several potential confounders (although as the exposure of blood group was genetic, and could be considered essentially randomised this changed little). As certain people with high-cholesterol may choose to treat the condition with lifestyle changes before considering drugs, we considered both self-reports and self-reports validated by treatments. Despite lacking laboratory cholesterol measurements in the E3N study, which is a major limitation, we considered self-reported dyslipidaemia requiring treatment validated against the use of lipid-lowering medications from a large drug-reimbursement database. Similarly, certain data such as BMI and smoking were based on self-reported data, but have been shown to be reliable ${ }^{29}$. In this cohort of well educated women, strong agreement has been observed between for example; self-report of hypertension and specific anti-hypertensive medications in other validation studies ${ }^{30}$. Whilst we are confident that the cases considered are true cases of dyslipidaemia, there is likely under reporting in the cohort from undiagnosed cases, which may weaken the observed associations. Other limitations include those common to all observational research; we cannot claim causality, there may be unmeasured confounding, and some participants may have misreported information. For 
example, unmeasured confounding likely explains that women with dyslipidaemia at baseline were less likely to smoke, and is possibly explained by health seeking behaviour (i.e. quitting smoking) as a result of a diagnosis of dyslipidaemia. Sample sizes in certain subgroups, for example AB women, were small. In particular, the E3N cohort is a well-educated population, with a low BMI, high levels of physical activity, and few current smokers. This means that the study may not be representative of the general population.

\section{Conclusion}

Non-O blood groups were associated with an increased risk of dyslipidaemia. In the whole cohort, the association was specifically attributed to the A-group. No interaction was observed with smoking status. People of the A-blood group should be considered at high risk for dyslipidaemia, which could present as a group that may benefit from early intervention on cholesterol levels.

\section{Data availability}

The datasets used and/or analysed during the current study are available from the corresponding author on reasonable request.

Received: 13 October 2020; Accepted: 11 June 2021

Published online: 21 July 2021

\section{References}

1. Kuo, P. T. Dyslipidemia and coronary artery disease. Clin. Cardiol. 17(10), 519-527. https://doi.org/10.1002/clc.4960171003 (1994).

2. Chapman, M. J. et al. Triglyceride-rich lipoproteins and high-density lipoprotein cholesterol in patients at high risk of cardiovascular disease: Evidence and guidance for management. Eur. Heart J. 32(11), 1345-1361. https://doi.org/10.1093/eurheartj/ehr112 (2011).

3. Hermida, N. \& Balligand, J.-L. Low-density lipoprotein-cholesterol-induced endothelial dysfunction and oxidative stress: The role of statins. Antioxid. Redox Signal. 20(8), 1216-1237. https://doi.org/10.1089/ars.2013.5537 (2014).

4. Giannitsi, S., Bougiakli, M., Bechlioulis, A. \& Naka, K. Endothelial dysfunction and heart failure: A review of the existing bibliography with emphasis on flow mediated dilation. JRSM Cardiovasc. Dis. 8, 2048004019843047. https://doi.org/10.1177/20480 04019843047 (2019).

5. Gillum, R. F. Blood groups, serum cholesterol, serum uric acid, blood pressure, and obesity in adolescents. J. Natl. Med. Assoc. 83(8), 682-688 (1991).

6. Garrison, R. J. et al. ABO blood group and cardiovacular disease: The Framingham study. Atherosclerosis 25(2-3), 311-318. https:// doi.org/10.1016/0021-9150(76)90036-8 (1976).

7. Medalie, J. H. et al. Blood groups and serum cholesterol among 10,000 adult males. Atherosclerosis 14(2), 219-229. https://doi. org/10.1016/0021-9150(71)90051-7 (1971).

8. Chen, Y. et al. Analysis of circulating cholesterol levels as a mediator of an association between ABO blood group and coronary heart disease. Circ. Cardiovasc. Genet. 7(1), 43-48. https://doi.org/10.1161/CIRCGENETICS.113.000299 (2014).

9. Wu, O., Bayoumi, N., Vickers, M. A. \& Clark, P. $\mathrm{ABO}(\mathrm{H})$ blood groups and vascular disease: a systematic review and meta-analysis: ABO groups and thrombosis. J. Thromb. Haemost. 6(1), 62-69. https://doi.org/10.1111/j.1538-7836.2007.02818.x (2007).

10. Dentali, F., Sironi, A., Ageno, W., Crestani, S. \& Franchini, M. ABO blood group and vascular disease: An update. Semin. Thromb. Hemost. 40(01), 049-059. https://doi.org/10.1055/s-0033-1363460 (2013).

11. Wu, O., Bayoumi, N., Vickers, M. A. \& Clark, $\mathrm{P} . \mathrm{ABO}(\mathrm{H})$ blood groups and vascular disease: A systematic review and meta-analysis. J. Thromb. Haemost. JTH 6(1), 62-69. https://doi.org/10.1111/j.1538-7836.2007.02818.x (2008).

12. Bruckert, E. et al. Relationship between smoking status and serum lipids in a hyperlipidemic population and analysis of possible confounding factors. Clin. Chem. 38(9), 1698-1705 (1992).

13. Gossett, L. K. et al. Smoking intensity and lipoprotein abnormalities in active smokers. J. Clin. Lipidol. 3(6), 372-378. https://doi. org/10.1016/j.jacl.2009.10.008 (2009).

14. Rigotti, N. A. \& Clair, C. Managing tobacco use: the neglected cardiovascular disease risk factor. Eur. Heart J. 34(42), 3259-3267. https://doi.org/10.1093/eurheartj/eht352 (2013).

15. Wakabayashi, I. \& Groschner, K. Age-dependent associations of smoking and drinking with non-high-density lipoprotein cholesterol. Metabolism 59(7), 1074-1081. https://doi.org/10.1016/j.metabol.2009.11.004 (2010).

16. Ambrose, J. A. \& Barua, R. S. The pathophysiology of cigarette smoking and cardiovascular disease. J. Am. Coll. Cardiol. 43(10), 1731-1737. https://doi.org/10.1016/j.jacc.2003.12.047 (2004).

17. El-Galaly, T. C. et al. Interaction between blood type, smoking and factor V Leiden mutation and risk of venous thromboembolism: a Danish case-cohort study: Letters to the Editor. J. Thromb. Haemost. 10(10), 2191-2193. https://doi.org/10.1111/j.1538-7836. 2012.04772.x (2012).

18. Clavel-Chapelon, F., E3N Study Group. Cohort profile: The french E3N cohort study. Int. J. Epidemiol. 44(3), 801-809, doi: https:// doi.org/10.1093/ije/dyu184 (2015).

19. Fournier, A. et al. Use of different postmenopausal hormone therapies and risk of histology- and hormone receptor-defined invasive breast cancer. J. Clin. Oncol. 26(8), 1260-1268. https://doi.org/10.1200/JCO.2007.13.4338 (2008).

20. Groot, H. E. et al. Genetically determined ABO blood group and its associations with health and disease. Arterioscler. Thromb. Vasc. Biol. 40(3), 830-838. https://doi.org/10.1161/ATVBAHA.119.313658 (2020).

21. Franchini, M., Mengoli, C., Bonfanti, C., Rossi, C. \& Lippi, G. Genetic determinants of extreme longevity: The role of ABO blood group. Thromb. Haemost. 115(2), 458-460. https://doi.org/10.1160/TH15-05-0379 (2016).

22. Capuzzo, E. et al. The relationship between ABO blood group and cardiovascular disease: results from the Cardiorisk program. Ann. Transl. Med. 4(10), 189. https://doi.org/10.21037/atm.2016.03.58 (2016).

23. Paré, G. et al. Novel association of ABO histo-blood group antigen with soluble ICAM-1: Results of a genome-wide association study of 6,578 women. PLoS Genet. 4(7), e1000118. https://doi.org/10.1371/journal.pgen.1000118 (2008).

24. Karakas, M. et al. A variant in the abo gene explains the variation in soluble E-selectin levels-results from dense genotyping in two independent populations. PLoS ONE 7(12), e51441. https://doi.org/10.1371/journal.pone.0051441 (2012).

25. Paterson, A. D. et al. Genome-wide association identifies the ABO blood group as a major locus associated with serum levels of soluble E-selectin. Arterioscler. Thromb. Vasc. Biol. 29(11), 1958-1967. https://doi.org/10.1161/ATVBAHA.109.192971 (2009).

26. Jenkins, P. V. \& O’Donnell, J. S. ABO blood group determines plasma von Willebrand factor levels: A biologic function after all?. Transfusion (Paris) 46(10), 1836-1844. https://doi.org/10.1111/j.1537-2995.2006.00975.x (2006).

27. Silbernagel, G. et al. High intestinal cholesterol absorption is associated with cardiovascular disease and risk alleles in ABCG8 and ABO. J. Am. Coll. Cardiol. 62(4), 291-299. https://doi.org/10.1016/j.jacc.2013.01.100 (2013). 
28. Paquette, M., Dufour, R. \& Baass, A. ABO blood group is a cardiovascular risk factor in patients with familial hypercholesterolemia. J. Clin. Lipidol. 12(2), 383-389.e1. https://doi.org/10.1016/j.jacl.2017.12.001 (2018).

29. Tehard, B., Liere, M. J. V., Nougué, C. C. \& Clavel-Chapelon, F. Anthropometric measurements and body silhouette of women. J. Am. Diet. Assoc. 102(12), 1779-1784. https://doi.org/10.1016/S0002-8223(02)90381-0 (2002).

30. Lajous, M. et al. Processed and unprocessed red meat consumption and hypertension in women. Am. J. Clin. Nutr. 100(3), 948-952. https://doi.org/10.3945/ajcn.113.080598 (2014).

\section{Acknowledgements}

We gratefully acknowledge the contribution of all the participants in the E3N study for their diligence and their answers. The authors have no conflicts of interest to declare.

\section{Author contributions}

C.J.M.- designed research, conducted research, analysed data, wrote paper. A.L.M.-conducted research, analysed data. A.F.— conducted research, analysed data. G.S.—-designed research, conducted research. M.C.B.R.primary responsibility for final content. All authors read and approved the final manuscript.

\section{Funding}

The E3N cohort is being carried out with the financial support of the "Mutuelle Générale de l'Education Nationale" (MGEN); European Community; French League against Cancer (LNCC); Gustave Roussy Institute (IGR); French Institute of Health and Medical Research (INSERM). This present study was also supported by the French Research Agency (Agence Nationale de la Recherche) via an "Investissement d'Avenir" grant (investment for the future grant, ANR-10-COHO-0006). CJM is supported by funding from the Federation Française de Cardiologie. The funders had no role in study design, data collection and analysis, decision to publish, or preparation of the manuscript.

\section{Competing interests}

The authors declare no competing interests.

\section{Additional information}

Supplementary Information The online version contains supplementary material available at https://doi.org/ 10.1038/s41598-021-94239-9.

Correspondence and requests for materials should be addressed to M.C.B.-R.

Reprints and permissions information is available at www.nature.com/reprints.

Publisher's note Springer Nature remains neutral with regard to jurisdictional claims in published maps and institutional affiliations.

Open Access This article is licensed under a Creative Commons Attribution 4.0 International License, which permits use, sharing, adaptation, distribution and reproduction in any medium or format, as long as you give appropriate credit to the original author(s) and the source, provide a link to the Creative Commons licence, and indicate if changes were made. The images or other third party material in this article are included in the article's Creative Commons licence, unless indicated otherwise in a credit line to the material. If material is not included in the article's Creative Commons licence and your intended use is not permitted by statutory regulation or exceeds the permitted use, you will need to obtain permission directly from the copyright holder. To view a copy of this licence, visit http://creativecommons.org/licenses/by/4.0/.

(C) The Author(s) 2021 